This is a preprint of an article accepted for publication in International Journal of Adaptive Control and Signal Processing, 2003, 17, 709-727

\title{
CONTROLLER PERFORMANCE ASSESSMENT IN SET POINT TRACKING AND REGULATORY CONTROL
}

\author{
N.F. Thornhill*, B. Huang ${ }^{+}$and S.L. Shah ${ }^{+}$ \\ "Department of Electronic and Electrical Engineering, University College London, Torrington Place, \\ London WC1E 7JE, UK \\ Tel: +442076793983 Fax: +442073889325 e-mail: n.thornhill@ee.ucl.ac.uk \\ ${ }^{+}$Department of Chemical and Materials Engineering, University of Alberta, Edmonton, T6G 2G6, \\ Canada
}

\begin{abstract}
Recent critiques of minimum variance benchmarking for single-input-single-output control loops have focused on the need for assessment of performance during set point changes and also on the need to pay attention to the movements in the manipulated variable. This paper examines factors that influence the minimum variance performance measure of a SISO control loop. It discusses the reasons why performance during set point changes differs from the regulatory performance during operation at a constant set point. The results demonstrate how regulatory performance is influenced by the nature of a disturbance, and that correlation of signals within a control loop can indicate whether the disturbance is random or deterministic. The paper is illustrated with simulated, experimental and industrial examples.
\end{abstract}

Keywords: Chemical industry; control loop performance; disturbance; fault diagnosis; minimum variance; plantwide disturbance; process control; process operation; set point control; regulation.

\section{INTRODUCTION}

Single-input-single-output control loop performance assessment has become an important technology in process operations. Many approaches are based upon the Harris index [1,2] which compares control loop performance against a minimum variance benchmark. The state of the art has been reviewed by Qin [3] and Harris et.al. [4].

Critiques of minimum variance benchmarking have recently come forward. Swanda and Seborg [5] and Isaksson and Horch [6] have shown it is desirable to have a separate assessment of performance during step changes. Seppala et. al., [7] discussed the influence of set point changes on the Harris index and demonstrated the benefits of a decomposition of the controller error into the components resulting from set point changes and a set-point detrended signal.
It is known, also, that minimum variance control may require excessively vigorous action of the manipulated variable and can lead to maintenance problems for actuators. There is thus an incentive to relax the minimum variance requirement. Kadali and Huang [8] and Grimble [9] have described benchmarks for Linear Quadratic Gaussian and Generalised Minimum Variance control that take into account the manipulated variable (i.e. the input into the controlled system) as well as the controlled variable in order to strike a balance between variability in the controlled variable and wear on the actuator. Xia and Howell [10] recently showed how an assessment of signal to noise ratio in the manipulated variable $(m v)$ can aid fault diagnosis in a system of interacting control loops. 
The contribution of this paper is to provide insights into control loop benchmarking of step changes and regulatory performance. The first outcome of the work is a demonstration that the minimum variance performance index determined during regulatory operation at a steady set point is not the same as that calculated during a set point change. The results show why separate benchmarks are needed for set point tracking and regulatory operation.

Regulatory and set point tracking performance differ because the presence of a disturbance affects the regulatory performance of a control loop. The presence of an external disturbance has previously been diagnosed by modeling or inspection of the crosscorrelation of the controller error, $y$, and a suspected disturbance variable [11-13]. However, in order to apply such methods it is useful to know first that a disturbance is present and an automated diagnostic step is required for that purpose. Correlation methods presented here are able to show when a disturbance is present.

A further contribution of the paper is to show how the nature of a disturbance influences the minimum variance performance index during regulatory operation. Control loops having a random disturbance are compared with loops influenced by a deterministic periodic disturbance. Correlations between the increments in the controller error or manipulated variable ( $\Delta y$ or $\Delta m v$ ), and $y$ or $m v$ are calculated, presented and explained. It is demonstrated that these correlations give a means for determination of the random or deterministic nature of the disturbance. Results from simulation and experimentation are reinforced by similar findings in an industrial data set.

The next section of the paper outlines the signal processing theory that is needed for analysis of the measurements. Section 3 presents a series of pilot-plant experiments involving set point changes and regulatory operation. A simulation of the pilot plant enabled additional trials of regulatory operation to be carried out that were infeasible to conduct experimentally. A distinctive feature of the simulations is that the sequence used to upset the simulated system was a periodic deterministic disturbance captured from the plant. The simulation results are therefore truly representative of real plant responses. Section 4 gives the results of analysis of the data from the experimental and simulation runs and an insight into their interpretation. Section 4 also describes similar findings with an industrial data set provided courtesy of Eastman Chemical Company. The paper ends with a conclusions section.

\section{THEORY}

This section introduces the classical approach to minimum variance benchmarking of a single-inputsingle-output control loop and presents the calculations for evaluation of the benchmark. Additionally, the correlation coefficient calculations used in disturbance diagnosis are introduced and a derivation given for the values of the correlation coefficients between controller error and its increments that are used in the diagnosis procedure.

The notation used is as follows: $p v$ is the process variable or controlled variable, $s p$ is the set point, $y$ is the controller error equal to $s p-p v$, and $m v$ is the manipulated variable. If a direct measurement of the $m v$ is not available then the controller output, op, is used instead.

\subsection{Minimum variance control during regulation}

As discussed in Huang and Shah [14] and Seppala et al, [7], the concept of the closed loop impulse underlies minimum variance benchmarking.

Figure 1 (left panel) shows the structure of a control loop subject to a disturbance $a$. The model for minimum variance benchmarking in regulatory operation uses the closed transfer function from $\{a(n)\}$ which is a white noise sampled data sequence to $\{y(n)\}$ :

$$
y(n)=-\frac{N}{1+q^{-d} T Q} a(n)
$$

In the above, $q^{-1}$ is the delay operator, $N$ is a filter, $Q$ is the controller, and $q^{-d} T$ is the controlled system which has a delay of $d$ sample intervals. The purpose of $N$ is to model general disturbances; for instance if the denominator of $N$ is a polynomial in $q^{-1}$ with roots close to the unit circle then the disturbance $v$ entering the control loop (see Figure 1) would exhibit autocorrelation over a large number of lags. Expression (1) can be transformed by polynomial division to:

$$
y(n)=H_{a}\left(q^{-1}\right) a(n)
$$

where $H_{a}\left(q^{-1}\right)$ is a polynomial whose coefficients are the impulse response coefficients. The coefficients $h_{a}(0) \ldots . . . h_{a}(d-1)$ are controller invariant while $h_{a}(d) h_{a}(d+1) \ldots$. can be set to zero by a minimum variance controller $Q_{m v}$. The minimum variance benchmark for a non-minimum variance controller is the ratio between the variance of the controller invariant part of the impulse response and the variance of the error signal $y$ : 


$$
\eta=\frac{\sigma_{m v}^{2}}{\sigma_{y}^{2}}=\frac{\sum_{i=0}^{d-1} h_{a}^{2}(i)}{\sum_{i=0}^{\infty} h_{a}^{2}(i)}
$$

The minimum variance controller $Q_{m v}$ depends upon the disturbance filter $N$ as well as on the controlled system $q^{-d} T$. Thus the $Q_{m v}$ for a control loop with a white noise disturbance when $N=1$ is not the same as for the case of integrated white noise with $N=\frac{1}{1-q^{-1}}$, and is different again when the disturbance is periodic. In the periodic case $N$ is a band-pass filter with a denominator polynomial having complex conjugate roots:

$$
N=\frac{1}{\left(1-q^{-1} \alpha\right)\left(1-q^{-1} \alpha^{*}\right)}
$$

\subsection{Minimum variance control for tracking of step changes}

If the set point is a step input then the model for the system is as shown in the right hand panel of Figure 1. An impulse sequence $\{x(n)\}=\left\{\begin{array}{lllll}1 & 0 & 0 & 0 & \ldots . .\end{array}\right\}$ is integrated by $N=\frac{1}{1-q^{-1}}$ to give a step signal at the $s p$. The transfer function is:

$$
y(n)=\frac{N}{1+q^{-d} T Q} x(n)
$$

which again can be expressed as a convolution of impulse response coefficients and the input sequence:

$$
y(n)=H_{u}\left(q^{-1}\right) x(n)
$$

In general, as highlighted by [7] the controller error $y$ may contain contributions both from disturbance and from set point changes. However, if there is no disturbance, or if the effect of the disturbance is negligible compared to the set point change, then the impulse response coefficients $h_{u}$ are the values of the controller error observed in the transient that follows a set point change. Thus the minimum variance index can be directly calculated from:

$$
\eta=\frac{\sigma_{m v}^{2}}{\sigma^{2} y}=\frac{\sum_{i=0}^{d-1} h_{u}{ }^{2}(i)}{\sum_{i=0}^{\infty} h_{u}{ }^{2}(i)}=\frac{\sum_{i=0}^{d-1} y^{2}(i)}{\sum_{i=0}^{\infty} y^{2}(i)}
$$

If the loop is under minimum variance control then the controller error becomes zero as soon as the dead time has elapsed and the ratio would be 1 .
As discussed earlier, the minimum variance controller for step inputs where $N=\frac{1}{1-q^{-1}}$ is not the same as the minimum variance controller for the random disturbance case or for the case when the disturbance is deterministic and periodic. Thus for an arbitrary controller $Q$ the calculated minimum variance benchmark $\eta$ is not, in general, expected to be the same for set point step changes, random disturbance and periodic deterministic disturbance.

\subsection{Calculation of $\eta$}

One method for calculation of $\eta$ is to directly estimate the impulse response coefficients for use in (3) by a signal processing technique such as the FCOR algorithm [14]. Desborough and Harris [2] suggested the following alternative procedure to calculate $\eta$. The controller error is decomposed into a $d$-step ahead prediction $\hat{y}$ and a residual $w$ by means of fitting of a $d$-step ahead ARMA model to the error sequence:

$$
y(n)=A\left(q^{-1}\right) y(n-d)+w(n)=\hat{y}(n)+w(n)
$$

The minimum variance is the variance of $w$, which is compared to the variance of the controller error to give the minimum variance benchmark:

$$
\eta=\frac{\sigma_{w}^{2}}{\sigma_{y}^{2}}
$$

The controller error of a loop under minimum variance control should have no $d$-step predictable component and therefore $\eta=1$ for a minimum variance controller. The calculations in this article followed [2] using equations (8) and (9).

\subsection{Correlation coefficients}

The correlation coefficient between two sampled data sequences $\left\{x_{1}(n)\right\}$ and $\left\{x_{2}(n)\right\}$ is estimated from $N_{s}$ samples as:

$$
r_{x_{1}, x_{2}}=\frac{1}{N_{s}} \sum_{n=1}^{N_{s}}\left(x_{1}^{\prime}(n) x_{2}^{\prime}(n)\right)
$$

where $x^{\prime}(n)=\frac{x(n)-\bar{x}}{\sigma_{x}}$, i.e. the sequences are meancentered and normalised to unit standard deviation.

Correlations between the increments in the controller error $(\Delta y)$ and the controller error $y$ or $m v\left(r_{\Delta y, y}\right.$ and $\left.r_{\Delta y, m v}\right)$ are likely to be different depending on the nature of the disturbance and thus have the potential to give a disturbance signature. Correlations $r_{\Delta m v, m v}$ between $m v$ and $m v$ movements (i.e. the increments in the $m v$ ) are also of interest. 
Random error: Assuming without loss of generality that the controller error is zero mean, then

$$
y^{\prime}(n)=\frac{y(n)}{\sigma_{y}}
$$

Increments in the controller error are calculated as:

$$
\Delta y(n)=y(n)-y(n-1)
$$

If the controller error sequence $\{y(n)\}$ is random uncorrelated noise then:

$$
\operatorname{Var}(\Delta y)=2 \times \operatorname{Var}(y)
$$

because the cross-term involving $\sum y(n) \times y(n-1)$ in the variance estimate is zero. Thus the standard deviation of the $\Delta y$ sequence is $\sqrt{2} \sigma_{y}$ and

$$
\Delta y^{\prime}(n)=\frac{y(n)-y(n-1)}{\sqrt{2} \sigma_{y}}
$$

If the controller error is random white noise the correlation coefficient between $\Delta y$ and $y$ is:

$$
\begin{aligned}
r_{\Delta y, y} & =\frac{1}{N_{s}} \sum_{n=1}^{N_{s}}\left(\frac{y(n)}{\sigma_{y}} \times \frac{y(n)-y(n-1)}{\sqrt{2} \sigma_{y}}\right) \\
& =\frac{\sigma_{y}^{2}}{\sqrt{2} \sigma_{y}{ }^{2}}-0=0.707
\end{aligned}
$$

Periodic error: Alternatively, if the controller error is periodic with period $T_{p}$ then the controller error sequence is sampled from $\sin \left(t \times 2 \pi / T_{p}\right)$. If $T_{p} \gg T$, where $T$ is the sampling interval, then $\Delta y$ is sampled from

$$
\Delta y=T \frac{2 \pi}{T_{p}} \cos \left(t \times 2 \pi / T_{p}\right)
$$

because

$$
d y / d t=\frac{2 \pi}{T_{p}} \cos \left(t \times 2 \pi / T_{p}\right)
$$

Thus if the controller error is periodic the correlation coefficient between $\Delta y$ and $y$ is:

$$
\begin{aligned}
r_{\Delta y, y} & =\frac{1}{N_{s}} \sum_{n=1}^{N_{s}}\left(\frac{\sin \left(n T \times 2 \pi / T_{p}\right)}{1 / \sqrt{2}} \times \frac{\cos \left(n T \times 2 \pi / T_{p}\right)}{1 / \sqrt{2} \times T \times 2 \pi / T_{p}}\right) \\
& \approx 0
\end{aligned}
$$

The result is close to zero because sine and cosine are orthogonal functions and it is exactly equal to zero if the $N_{s}$ terms in the sum capture a whole number of complete cycles of the periodic signal. The $1 / \sqrt{2}$ factors arise because the r.m.s. value (the standard deviation) of a unit amplitude sine wave is $1 / \sqrt{2}$.

These numerical results will be exploited later in the diagnosis of the nature of a disturbance.

Mixed error: This sub-section will determine the value of $r_{\Delta y, y}$ when the controller error is sampled from a periodic deterministic component plus a random component. The random component is $w$ having unit variance and the deterministic component is sampled from a sine wave having period $T_{p}$ and amplitude $\alpha$.

If $\alpha=\sqrt{2}$ then the variance of the deterministic component is unity because the r.m.s value of a sine function is $1 / \sqrt{2}$ :

$$
y(n)=w(n)+\alpha \sin \left(n T \times 2 \pi / T_{p}\right)
$$

The standard deviation of $y(n)$ calculated from the samples is approximately $\sqrt{\left(1+\alpha^{2}\right)}$ and is exactly that value if the calculation captures a complete number of cycles of the sine wave.

If $T_{p} \gg T$ the controller error increments are:

$$
\begin{aligned}
\Delta y(n) & =y(n)-y(n-1) \\
& =w(n)-w(n-1)+\alpha T \frac{2 \pi}{T_{p}} \cos \left(n T \times 2 \pi / T_{p}\right)
\end{aligned}
$$

and the standard deviation of this sequence is

$$
\sqrt{2+\left(\alpha T \frac{2 \pi}{T_{p}}\right)^{2}} \approx \sqrt{2}
$$

Thus

$$
\begin{aligned}
r_{\Delta y, y}=\frac{1}{N_{s}} \sum_{n=1}^{N_{s}}\left(\frac{\left(w(n)+\alpha \sin \left(n T \times 2 \pi / T_{p}\right)\right)}{\sqrt{\left(1+\alpha^{2}\right)}} \times\right. & \left.\frac{\left(w(n)-w(n-1)+\alpha T \frac{2 \pi}{T_{p}} \cos \left(n T \times 2 \pi / T_{p}\right)\right)}{\sqrt{2}}\right) \\
& \approx \frac{1}{\sqrt{2} \sqrt{\left(1+\alpha^{2}\right)}}
\end{aligned}
$$

Again, the equality is exact if the sampled data sequence captures a complete number of cycles of the periodic sequence.

If $\alpha=\sqrt{2}$ as when the random and periodic disturbances have equal variance then $r_{\Delta y, y}=0.41$.

Therefore $r_{\Delta y, y}=0.41$ will be used as a threshold to determine whether the controller error is predominantly random. 
Correlation of $\Delta m v$ and $m v$ : The same comments as above concerning the values in the random, periodic and mixed case apply also to $r_{\Delta m v, m v}$.

\section{METHODS}

This section presents experimental measurements from a pilot scale stirred tank reactor and a series of simulations of the same stirred tank. A set of step test experiments were carried out on a level control loop and a temperature control loop. Data were collected during the transient step response and also during normal running (regulatory operation) once the transients had died away.

A simulation was created that used heat and mass balance together with valve, instrument and heat transfer characteristics measured during calibration of the plant. Disturbances in the simulation were provided as numerical sequences of the real noise collected during open loop testing of the plant. The simulations explored the relative influence of a random noise disturbance and a periodic deterministic disturbance when applied together in varying ratios.

\subsection{Step tests}

The process schematic is shown in Figure 2 for a continuous stirred tank reactor in the Computer Process Control group of the University of Alberta, Department of Chemical and Materials Engineering. Computer control of the process was achieved using Simulink and the Real Time Toolbox of MATLAB (The Mathworks, Natick, MA) interfaced with the plant. Actuator demands calculated within Simulink were sent to the plant as $4-20 \mathrm{~mA}$ signals, and 4-20 mA signals from the instruments on the plant were sent to Simulink for calculation of control actions. A cascade configuration onto cold water flow was implemented for level control, as shown in Fig 2, and the temperature control used proportional plus integral control of the steam valve. A benefit of the configuration is its flexible access to the plant inputs. For instance, it is possible to add a known disturbance to the output of a controller so that the valve receives a signal comprising the controller demand plus a disturbance. A real process disturbance could be applied by bubbling compressed air through the vessel.

Step test sequences numbered 1 to 8 (see Table 1) were applied to the plant for assessment of set point step changes giving the responses shown in the left hand panels of Figure 3. Each step test used different settings of the proportional plus integral controller. Data sequences from regulatory operation at a steady set point were also captured using the same controller settings, as shown in the middle panels of Figure 3. The noise levels in the temperature loop were much higher than in the level loop. The right hand panels show the autocovariance functions for the data from regulatory operation where it can be seen that the level measurement had a long range periodic autocovariance while the temperature disturbance was more random having only short range correlation.

\subsection{Collection of disturbance sequences}

Figure 4(a) (upper left panel) shows an open loop measurement from a steady level signal when compressed air bubbles were blown into in the tank while Figure 4(b) (lower left panel) is the variation in the cold water flow measurement when the valve demand signal was held constant. These data were reused as disturbance inputs for the simulation experiments to be described in section 3.3. The disturbance from compressed air bubbles is random, as can be seen from its negligible autocovariance function (upper right panel). The cold water disturbance is deterministic because it is periodic and predictable, having a distinctive oscillatory feature at about 40 samples per cycle. It has long range autocovariance.

\subsection{Disturbance tests}

Two experimental disturbance tests were conducted on the level loop in the pilot plant and additional disturbance tests were conducted in a series of simulated runs. The controller settings were the same in each case, and were those from Test 2 shown in Table 1. Tests 9 and 10 described in Table 2 were applied to the plant for 500s. In Test 10 the disturbance shown in Figure 4(b) was applied to the cold water valve demand. To achieve that, the disturbance was added to the controller output and the sum of the two sent to the $\mathrm{D} / \mathrm{A}$ converter to create the analogue signal to drive the valve. The flow disturbance sequence was amplified by a factor of 10 is so that its effects would dominate the natural process disturbance.

Simulation Tests 11 to 15 were run for 2000s. In those cases there was no natural process noise and the flow disturbance was applied without amplification. Figure 5 shows the time trends of $y$ and $m v$

\subsection{Industrial data}

Measurements from an industrial plant were provided courtesy of the Eastman Chemical Company, Kingsport, TN. A deterministic, periodic disturbance caused by limit cycling of a sticking control valve had propagated to many locations in the plant. The upper panels in Figure 6 show the time trends of controller error and manipulated variable for several flow and level measurements from the plant taken before the sticking control valve had been diagnosed and the lower panels show the same measurements after the valve was repaired. The periodic disturbance is absent in the lower plots.

\subsection{Data analysis}

Performance indexes: Controller performance indexes $\eta$ were calculated. For the step changes in Tests 1 to 8 
$\eta$ was computed using data sets starting $50 s$ before the step and continuing for $250 \mathrm{~s}$ after the step, a total of 301 samples. The results determined from application of (8) and (9) were compared to the results of direct calculation using (7). The calculations require the loop time delays which for the level and temperature loops were $2 \mathrm{~s}$ and $8 \mathrm{~s}$ respectively.

The regulatory performances for Tests 1 to 8 were determined from 2000 samples from regulatory operation using equations (8) and (9). Performance indexes were also determined for regulatory operation in Tests 9 to 15 and for the industrial data.

Correlation coefficients: Correlation coefficients $r_{\Delta y, y}$, $r_{\Delta y, m v}$ and $r_{\Delta m v, m v}$ were determined for all the experiments and simulations in order to find out if these correlation coefficients varied in a systematic manner as the nature of the disturbance changed from random to deterministic. Similar calculations were done for the industrial data.

\section{RESULTS}

\subsection{Plant experiments}

Figure 3 shows set point step tests for various controller tuning settings of the level-flow cascade control loop and the temperature loop. The $\eta$ results are in Table 1 together with the directly-calculated minimum variance measure.

Fig 7 (left panel) compares results from direct calculation using (7) and the $\eta$ calculated from (8) and

(9) during the step change. The closeness of the results to the unit gradient line shows that two methods gave almost the same estimate of the minimum variance performance index for a set point step change. The right hand panel of Figure 7 , however, shows that $\eta$ for the steps response was not related to the $\eta$ during regulatory operation. The next section will explain this finding.

Figure 8 gives a visual comparison of the numerical results by means of bar charts. The right hand panels show that $r_{\Delta m v, m v}$ during regulatory operation was related to the $\eta$ for set point tracking. It has a low value in loops with a slow and poorly damped set point performance (Tests 1-4 and 8) and was large when the loop had aggressive set point tracking performance (Tests 5 and 7). Therefore the loops closer to minimum variance set point tracking control had more random $m v$ movements during regulatory operation, while those with a slow and poorly damped set point performance had smoother and more deterministic $m v$ movements. These features can be seen in lower middle panel of Figure 3 where the most vigorous $m v$ action is in Test 5 and 7.
The value of $r_{\Delta m v, m v}$ was not, however, related to $\eta$ during regulatory operation. Tests 5 and 6 show that $r_{\Delta m v, m v}$ could be reduced to below 0.41 and thus changed from random to deterministic without much change to the $\eta$ for regulatory operation. There would be less wear on the actuator when the $m v$ is smoother and $r_{\Delta m v, m v}$ is smaller. The results found here reinforce the proposals in [8-10], and elsewhere in this Special Issue, where the need for an enhanced controller performance measure that pays attention to the $m v$ has been identified.

\subsection{Disturbances}

The following analysis explores the behaviour of the performance index and other quantities when the disturbances shown in Figure 4 were present in varying ratios in the level loop. Table 3 shows the performance indexes from regulatory operation and correlation coefficients for the regulatory operation of the plant (Tests 1-10) and simulated runs (Tests 11 to 15). Observations are:

- The performance index $\eta$ depended upon the nature of the disturbance;

- There was a high correlation between the dominant disturbance and the controller error $y$;

- The correlation coefficients $r_{\Delta y, y}$ and $r_{\Delta y, m v}$ depended on the nature of the disturbance;

- The correlation coefficient $r_{\Delta m v, m v}$ was not large for any of the experimental or simulation runs for the level loop (Tests 1-4, 9-15).

\subsection{Discussion}

The upper left hand panel of Figure 8 shows that the tests dominated by deterministic flow disturbance (10, $14,15)$ had a low $\eta$ while those dominated by the random bubble disturbance $(9,11,12)$ had a high $\eta$. Thus the $\eta$ value during regulatory control responds to the nature of the disturbance even though the controller tuning setting did not change. Test 13 with equal weights for the random and deterministic disturbances had an intermediate value of $\eta$.

The behaviour of the correlation coefficient $r_{\Delta y, y}$ is similar to that of the $\eta$ for regulatory operation. Since $r_{\Delta y, y}$ gives a test of the randomness of controller error, the results confirm that high values of $\eta$ in regulatory operation are achieved when the controller error is random.

As expected ([11-13]), there is strong correlation between the dominant disturbance and the process variable (Table 3, columns 5 and 6), and therefore with $y$. The correlation was not perfect because of the loop dynamics. For instance, first order lag dynamics introduce a phase shift and there may be other noise 
present. The finding nevertheless shows that the nature of a disturbance may be inferred from an inspection of $y$.

The data from the level loop gave systematic trends in $r_{\Delta y, y}$ and $r_{\Delta y, m v}$, as shown in Figure 9 which plots $r_{\Delta y, y}$ on the horizontal axis and $r_{\Delta y, m v}$ on the vertical axis. Cases with random disturbance appeared at the lower right side of the plot, cases with a periodic deterministic disturbance were in the left hand top corner. The explanation of the relationship is explored in the Analysis section below. Also shown in Figure 9 are the plant runs, Test 9 with bubbles present on the right and Test 10 with deterministic flow noise disturbance on the left.

Other plant results for the level loop (Tests 1-4) were clustered close to one another and also lay near the curve. Therefore those plant runs were diagnosed as having some periodic deterministic disturbance but not as much as in Test 10. This is a correct finding because the level loop was subject to the natural cold water flow disturbance in Tests 1 to 4 whereas in Test 10 the deterministic flow disturbance was magnified by a factor of ten.

\subsection{Analysis}

Correlation of $\Delta y$ with $y$ : The controller error $y$ is somewhat correlated with the true disturbance because the $p v$ is correlated with the disturbance. Thus $y$ has a random component if the disturbance is random and it is to be expected that random fluctuations in the controller error $y$ will show a $r_{\Delta y, y}$ correlation of up to 0.707 , as demonstrated in equation 15 . A correlation coefficient of 0.65 , close to the maximum expected value, was observed in Test 11 which had the largest random disturbance. The tests with predominantly random disturbance $(9,11,12)$ all had values of $r_{\Delta y, y}$ above the threshold value of 0.41 that was derived in section 2.4.

For a deterministic periodic disturbance there should be little correlation between $\Delta y$ and $y$ (equation 17). The expectation was confirmed by Tests 10,14 and 15 with deterministic disturbances which had $r_{\Delta y, y}$ values below the threshold of 0.41 . Test 13 that had equal amounts of the two disturbances had an $r_{\Delta y, y}$ value of 0.39 , close to the threshold.

Correlation of $\Delta y$ with $m v$ : The reason why $\Delta y$ correlated with $m v$ in the deterministic periodic cases (Tests 10, 14 and 15) is because of the structure and dynamics of the level control loop. The deterministic disturbance was a disturbance to the flow valve and therefore affected $m v$ directly. The controlled process (the tank) has integrating dynamics which means the $p v$ and also the controller error $y$ have time trends that are similar to the integral of $m v$. The time trend of $\Delta y$ is thus similar to the trend of $m v$ because $\Delta y$ is the numerical derivative of $y$. As a result the correlation coefficient $r_{\Delta y, m v}$ must be strong in the case of the deterministic flow disturbance signal.

The random disturbance caused by compressed air bubbles, by contrast, acted directly on the level measurement and the controller error is therefore also random. The $m v$ is derived from the random signal through the proportional plus integral controller and there is no correlation of $\Delta y$ with $m v$ in that case.

The argument that the correlation will be small for a random disturbance would not hold true for a controlled system having a P-only controller. Therefore it is not possible to generalise the use of $r_{\Delta y, m v}$. For instance, the value of $r_{\Delta y, y}$ for regulatory operation in Test 5 was found to be 0.56 , revealing the random nature of the controller error. However, the controller in Test 5 has weak $I$-action and the $m v$ is similar to the (random) controller error $y$, as can be seen in Figure 3 . As a result $r_{\Delta y, m v}$ is similar to $r_{\Delta y, y}$ and was found to be 0.52 , not small or close to zero.

Generalisation: The above observations show that values above 0.41 in the correlation coefficient $r_{\Delta y, y}$ means the controller error is random. The opposite applies to $r_{\Delta m v, m v}$. If the value is lower than 0.41 it means the $m v$ is moving more smoothly and less randomly. For controller performance purposes, the following targets are suggested:

- $r_{\Delta y, y}$ should be above 0.41 to ensure the controller error is random;

- $r_{\Delta m v, m v}$ should be below 0.41 to ensure that $m v$ movements are not too aggressive.

The discussion in the previous sub-section also indicates that use of the $r_{\Delta y, m v}$ correlation is not generalisable because it depends upon the structure of the control loop.

\subsection{Industrial example}

The upper panel of Figure 10 shows bar charts for the minimum variance control loop performance index $\eta$ before and after maintenance work in the plant which cured a plant-wide disturbance. The controller performance index was calculated for regulatory operation only since there were no step changes of set point in the data set. The lower panels in Figure 10 show $r_{\Delta y, y}$ and $r_{\Delta m v, m v}$ before and after maintenance. The correlation coefficient $r_{\Delta y, m v}$ is not presented because, as discussed above, it cannot be generalised.

The upper panels of Figure 6 show the presence of a deterministic disturbance with a period of 330 sampling intervals. It is visibly present in the controller errors of 
loops 16 and 19, and in all the $m v$ trends except for 17 . The lower panels show that the deterministic disturbance disappeared from both the controller error and $m v$ time trends after the repair.

The following observations can be made:

- The controller performance index $\eta$ for regulatory operation improved in the loops numbered 16, 18 and 19 after the repair of the faulty valve. The performance of loop 19, though greatly improved, was still not good;

- The $r_{\Delta y, y}$ values for loops 16 and 18 rose above the 0.41 threshold after the repair. Therefore after the repair those controller errors were predominantly random. The controller error for loop 19 was not random after the repair showing that the loop remains subject to an unknown deterministic disturbance. This may be no more than the slow meanderings about the set point that can be observed in the lower left panel of Figure 6;

- The performance of loop 17 was high and its $r_{\Delta y, y}$ value was high both before and after the repair. The result shows that loop 17 is well tuned, has a random controller error and was not influenced by the disturbance;

- The performance of loop 20 was intermediate but its $r_{\Delta y, y}$ value was low both before and after the repair. The result shows that the controller error of loop 20 was influenced by a second disturbance which did not go away after the repair. Figure 6 (left panels) shows the second disturbance has the form of frequent spiky transient effects with a decay time constant of about 8-10 samples;

- The values of $r_{\Delta m v, m v}$ for all loops were below the 0.41 threshold both before and after the repair suggesting that the $m v$ movements are not overly aggressive.

\section{CONCLUSIONS}

The paper has used plant experimentation, simulation and an industrial example to demonstrate practical issues arising in the interpretation of the minimum variance control loop performance index $\eta$ described by Harris [1] and Desborough and Harris [2].

Theory shows that for an arbitrary controller $Q$ the calculated minimum variance benchmark $\eta$ is not the same for a step change in the set point as for regulatory operation. The work reported here gives practical demonstrations of those observations and in particular shows that the performance during regulatory control depends on the nature of the disturbance. The value of $\eta$ during a step change in set point is not related to $\eta$ during regulatory operation $\eta$. In regulatory operation $\eta$ was low in the case of a deterministic periodic disturbance and high if the disturbance was random even though the controller tuning did not change.

As reported elsewhere [11-13] the controller error $y$ is somewhat correlated with the disturbance affecting the loop, so the nature of the disturbance may be inferred from $y$. The value of the correlation coefficient $r_{\Delta y, y}$ between increments in the controller error $(\Delta y)$ and $y$ reveals the nature of the controller error. If $r_{\Delta y, y}$ is above 0.41 the controller error is predominantly random and high values of $\eta$ in regulatory operation are achieved.

The need to reduce movements in the manipulated variable, $m v$, has also been considered. The converse comments apply $r_{\Delta m v, m v}$. If the value is lower than

0.41 it means the $m v$ is moving more smoothly and less randomly. For controller performance purposes, the following generic targets are suggested:

- $r_{\Delta y, y}$ should be above 0.41 to ensure the controller error is random;

- $r_{\Delta m v, m v}$ should be below 0.41 to ensure that $m v$ movements are not too aggressive.

It was demonstrated that $r_{\Delta m v, m v}$ for regulatory operation is related $\eta$ during a step change and not to $\eta$ during regulatory operation. The penalty for reducing $r_{\Delta m v, m v}$ is that the set point step change response becomes less aggressive and takes longer to settle.

The correlation $r_{\Delta y, m v}$ between $\Delta y$ and $m v$ was also examined. There were strong systematic trends within a given control loop, but the value of $r_{\Delta y, m v}$ was found to be dependent upon the structure and tuning of the control loop and therefore not generalisable.

The findings from simulation and pilot scale were observed also in an industrial data set. The success of the industrial study suggests that the new correlations $r_{\Delta y, y}$ and $r_{\Delta m v, m v}$ are generic and have a useful application in the performance assessment of SISO control loops.

\section{REFERENCES}

1. Harris, T.J. Assessment of control loop performance, Can. J. Chem. Eng. 1989; 67: 856-861.

2. Desborough, L., Harris, T.J. Performance assessment measures for univariate feedback control, Can. J. Chem. Eng. 1992; 70: 1186-1197.

3. Qin, S.J. Control performance monitoring - a review and assessment, Comput. Chem. Engng. 1998; 23: 173-186.

4. Harris, T.J., Seppala, C.T., Desborough, L. A review of performance monitoring and assessment techniques for univariate and multivariate control systems, Journal of Process Control 1999; 9: 1-17. 
5. Swanda, A.P., Seborg, D.E. Controller performance assessment based on setpoint response data. Proc. 1999 American Control Conf. 1999; 3863-3867.

6. Isaksson, A.J., Horch, A., Dumont, G.A. Event-triggered deadtime estimation from closed-loop data, Proc. 2001 American Control Conf. 2001; 3280-3285.

7. Seppala, C.T., Harris, T.J., Bacon, D.W., Time series methods for dynamic analysis of multiple controlled variables, Journal of Process Control, 2002; 12: 257 276.

8. Kadali R., Huang B. Controller performance analysis with LQG benchmark obtained under closed loop conditions, ISA Transactions 2002; 41: 521-537.

9. Grimble, M.J. Controller performance benchmarking and tuning using generalised minimum variance control, Automatica 2002; 38: 2111-2119.

10. Xia, C., Howell, J. Loop status monitoring and fault localisation, Journal of Process Control, 2003; in press: doi:10.1016/S0959-1524(02)00123-3.

11. Desborough, L., Harris T.J. Performance assessment measures for univariate feedforward-feedback control, Canadian Journal of Chemical Engineering, 1993; 71: 605-616.

12. Kozub, D.J., Garcia, C.E. Monitoring and diagnosis of automated controllers in the chemical process industries, AIChE Annual Meeting 1993; St Louis.

13. Stanfelj, N., Marlin T.E., MacGregor, J.F. Monitoring and diagnosing process control performance: The single loop case, Industrial Engineering Chem. Research, 1993; 32: $301-314$.
14. Huang, B., and Shah, S.L., Performance Assessment of Control Loops: Theory and applications, SpringerVerlag, 1999; ISBN: 1-85233-639-0.

\section{ACKNOWLEDGEMENTS}

The authors are grateful for the support of the Natural Science and Engineering Research Council (Canada), Matrikon (Edmonton, Alberta) and the Alberta Science and Research Authority through the NSERC-MatrikonASRA Industrial Research Chair in Process Control. The authors would like to thank John W. Cox and Michael A. Paulonis of the Eastman Chemical Company, Kingsport, TN for providing the industrial data. Thanks also to M.A. Shoukat Choudhury and R. Bushan Gopaluni for help with conducting the experimental runs for the study. 


\begin{tabular}{llccccc} 
Test description & $\begin{array}{c}\text { controller } \\
\text { gain } K\end{array}$ & $\begin{array}{c}\text { integral } \\
\text { time, } \tau_{i}\end{array}$ & $\begin{array}{c}\text { Direct min. var } \\
\text { calculation }\end{array}$ & $\begin{array}{c}\eta \text { during } \\
\text { step }\end{array}$ & $\begin{array}{c}\eta \text { in regulatory } \\
\text { operation }\end{array}$ \\
\hline 1 & step tests and & 2 & 40 & 0.20 & 0.23 & 0.20 \\
2 & regulatory operation & 2 & 20 & 0.19 & 0.22 & 0.30 \\
3 & of level loop & 2 & 13 & 0.18 & 0.21 & 0.30 \\
4 & & 1 & 10 & 0.09 & 0.11 & 0.18 \\
\hline 5 & step tests and & 6 & 60 & 0.74 & 0.76 & 0.98 \\
6 & regulatory operation & 3 & 30 & 0.47 & 0.52 & 0.96 \\
7 & of temperature & 3 & 15 & 0.61 & 0.67 & 0.99 \\
8 & loop & 1 & 5 & 0.34 & 0.41 & 0.91 \\
\hline
\end{tabular}

Table 1. Summary of performance results from experimental step and regulatory operation tests. The controller transfer function is $K\left(1+\frac{1}{s \tau_{i}}\right)$.

Test description

Disturbance

\begin{tabular}{cll}
\hline 9 & Compressed air bubbled through tank & real \\
10 & Flow disturbance $\times 10$ added to cold water valve demand & Fig 4(b) \\
11 & Bubble noise added as disturbance to level in simulation & Fig 4a(a) \\
$12-14$ & 11 and 15 in various combinations & $4(\mathrm{a})$ and 4(b) \\
15 & Flow disturbance added to cold water valve demand in simulation & Fig 4(b) \\
\hline
\end{tabular}

Table 2. Description of disturbance runs on the pilot plant and in simulation. These tests used the level loop with the same controller settings as for Test 2 .

\begin{tabular}{|c|c|c|c|c|c|c|c|}
\hline \multicolumn{2}{|c|}{ description } & \multirow[t]{2}{*}{$\eta$} & \multirow[t]{2}{*}{$r_{\Delta y, y}$} & \multirow[t]{2}{*}{$r_{\Delta y, m v}$} & \multirow[t]{2}{*}{$r_{\Delta m v, m v}$} & \multirow[t]{2}{*}{$r_{p v, b u b b l e s}$} & \multirow[t]{2}{*}{$r_{p v, \text { flow }}$} \\
\hline & pilot plant runs & & & & & & \\
\hline 1 & level loop - regulatory operation & 0.20 & 0.25 & 0.28 & 0.23 & - & - \\
\hline 2 & level loop - regulatory operation & 0.30 & 0.23 & 0.29 & 0.19 & - & - \\
\hline 3 & level loop - regulatory operation & 0.30 & 0.25 & 0.25 & 0.18 & - & - \\
\hline 4 & level loop - regulatory operation & 0.18 & 0.31 & 0.16 & 0.22 & - & - \\
\hline 5 & temp loop - regulatory operation & 0.98 & 0.56 & 0.51 & 0.52 & - & - \\
\hline 6 & temp loop - regulatory operation & 0.96 & 0.46 & 0.27 & 0.25 & - & - \\
\hline 7 & temp loop - regulatory operation & 0.99 & 0.57 & 0.55 & 0.51 & - & - \\
\hline 8 & temp loop - regulatory operation & 0.91 & 0.51 & 0.21 & 0.16 & - & - \\
\hline 9 & bubble disturbance & 0.85 & 0.64 & 0.05 & 0.22 & - & - \\
\hline 10 & flow disturbance & 0.03 & 0.11 & 0.76 & 0.15 & - & 0.76 \\
\hline & $\underline{\text { simulation runs }}$ & & & & & & \\
\hline 11 & bubbles only & 0.93 & 0.65 & 0.03 & 0.14 & 0.96 & - \\
\hline 12 & bubble dominated & 0.91 & 0.57 & 0.05 & 0.12 & 0.90 & 0.03 \\
\hline 13 & equal effects & 0.62 & 0.39 & 0.15 & 0.10 & 0.60 & 0.38 \\
\hline 14 & flow disturbance dominated & 0.12 & 0.20 & 0.38 & 0.10 & 0.25 & 0.59 \\
\hline 15 & flow disturbance only & 0.002 & 0.09 & 0.99 & 0.10 & - & 0.70 \\
\hline
\end{tabular}

Table 3. Performance index and correlation coefficients for pilot plant and simulated runs. 


\begin{tabular}{llcccccccc} 
& & \multicolumn{3}{c}{ before } & & \multicolumn{3}{c}{ after } \\
\cline { 3 - 4 } \cline { 7 - 9 } & description & $\eta$ & $r_{\Delta y, y}$ & $r_{\Delta m v, m v}$ & & & $r_{\Delta y, y}$ & $r_{\Delta m v, m v}$ \\
\hline 16 & industrial level loop & 0.21 & 0.25 & 0.19 & & 0.66 & 0.51 & 0.39 \\
17 & industrial flow loop & 0.91 & 0.55 & 0.10 & & 0.93 & 0.58 & 0.10 \\
18 & industrial flow loop & 0.63 & 0.30 & 0.17 & & 0.96 & 0.60 & 0.30 \\
19 & industrial level loop & 0.03 & 0.10 & 0.07 & & 0.32 & 0.29 & 0.05 \\
20 & industrial flow loop & 0.75 & 0.22 & 0.08 & & 0.70 & 0.22 & 0.05 \\
\hline
\end{tabular}

Table 4. Performance index for regulatory operation and correlation coefficients for industrial data before and after maintenance of a sticking valve. 

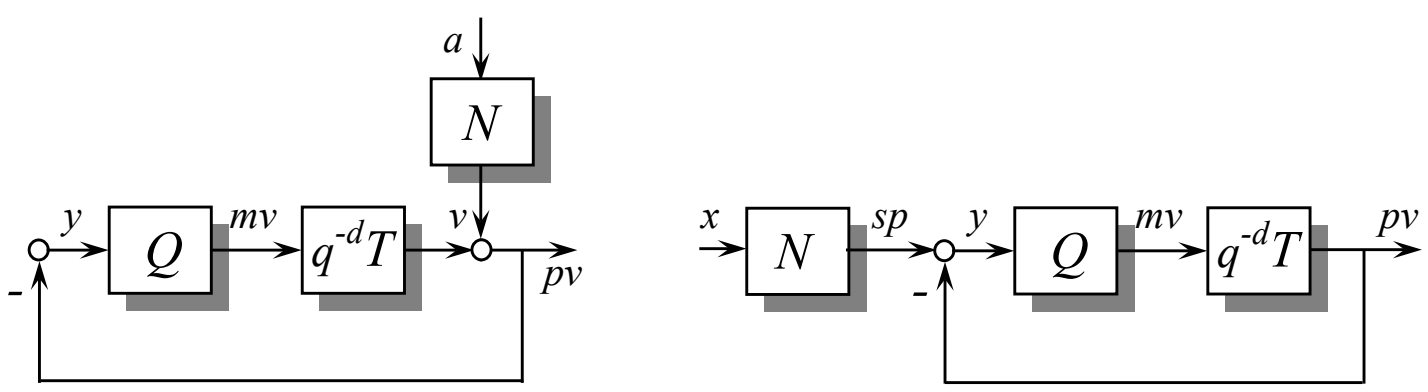

Figure 1. Block diagrams for analysis of the effects of disturbance and set point changes 


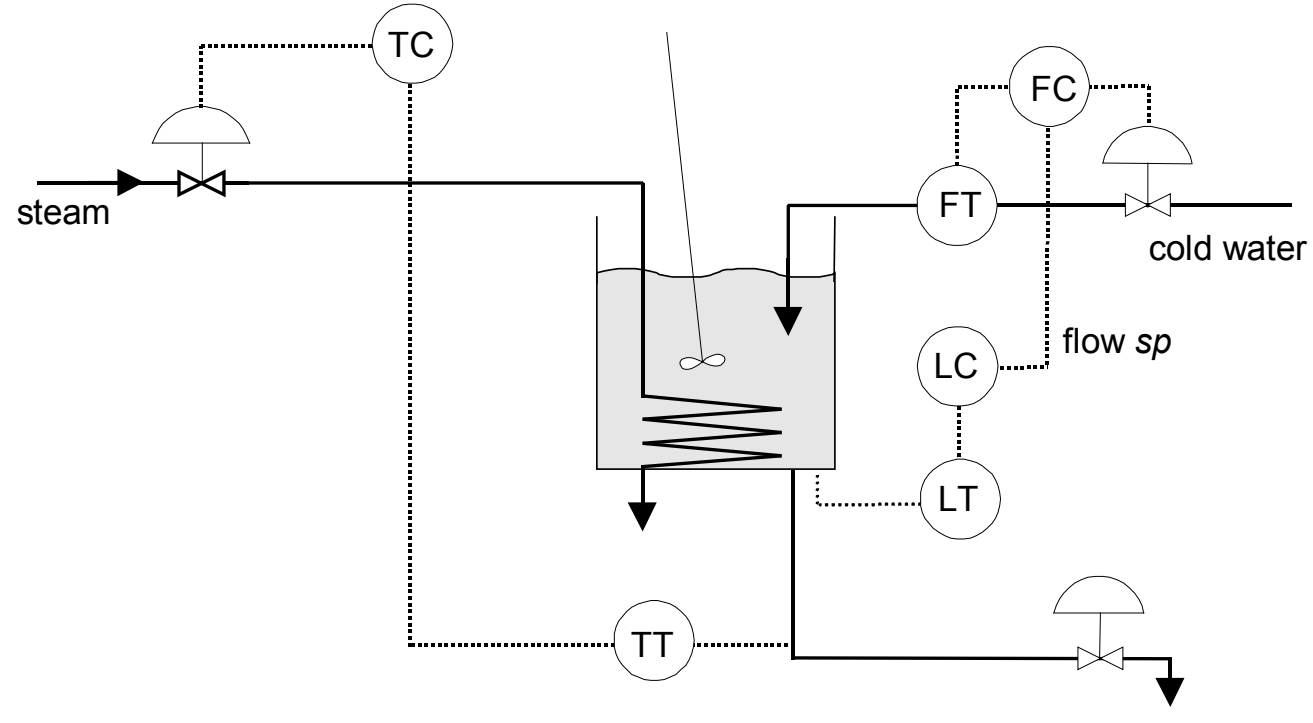

Figure 2. Pilot plant schematic 

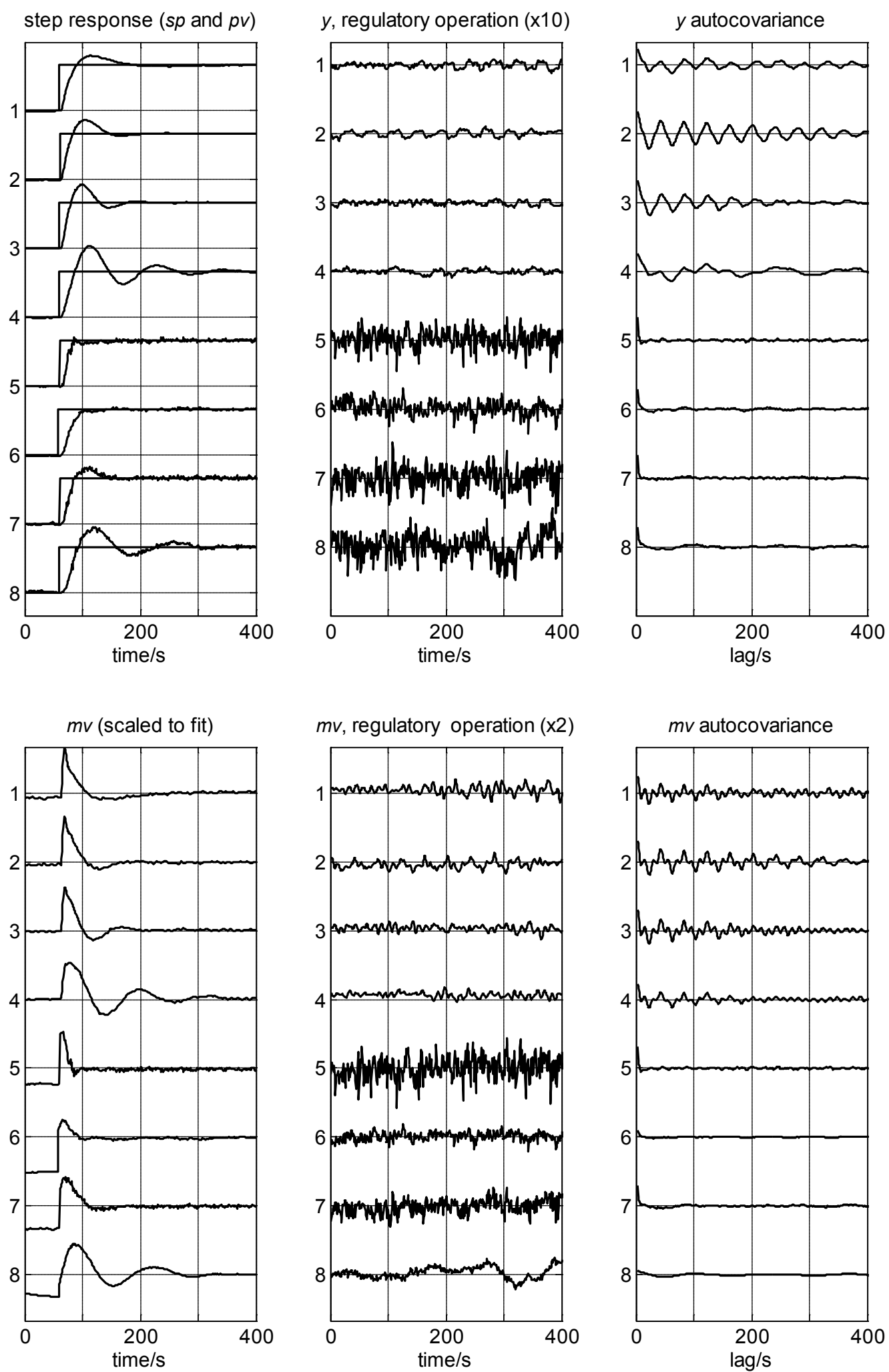

Figure 3. Pilot plant Tests 1 to 8 . Left panels: Responses to $2 \mathrm{~mA}$ step change in set point. Middle panels: controller error $y$ during normal running, magnified compared to left panel; Right panels: Autocovariance functions during normal running. 

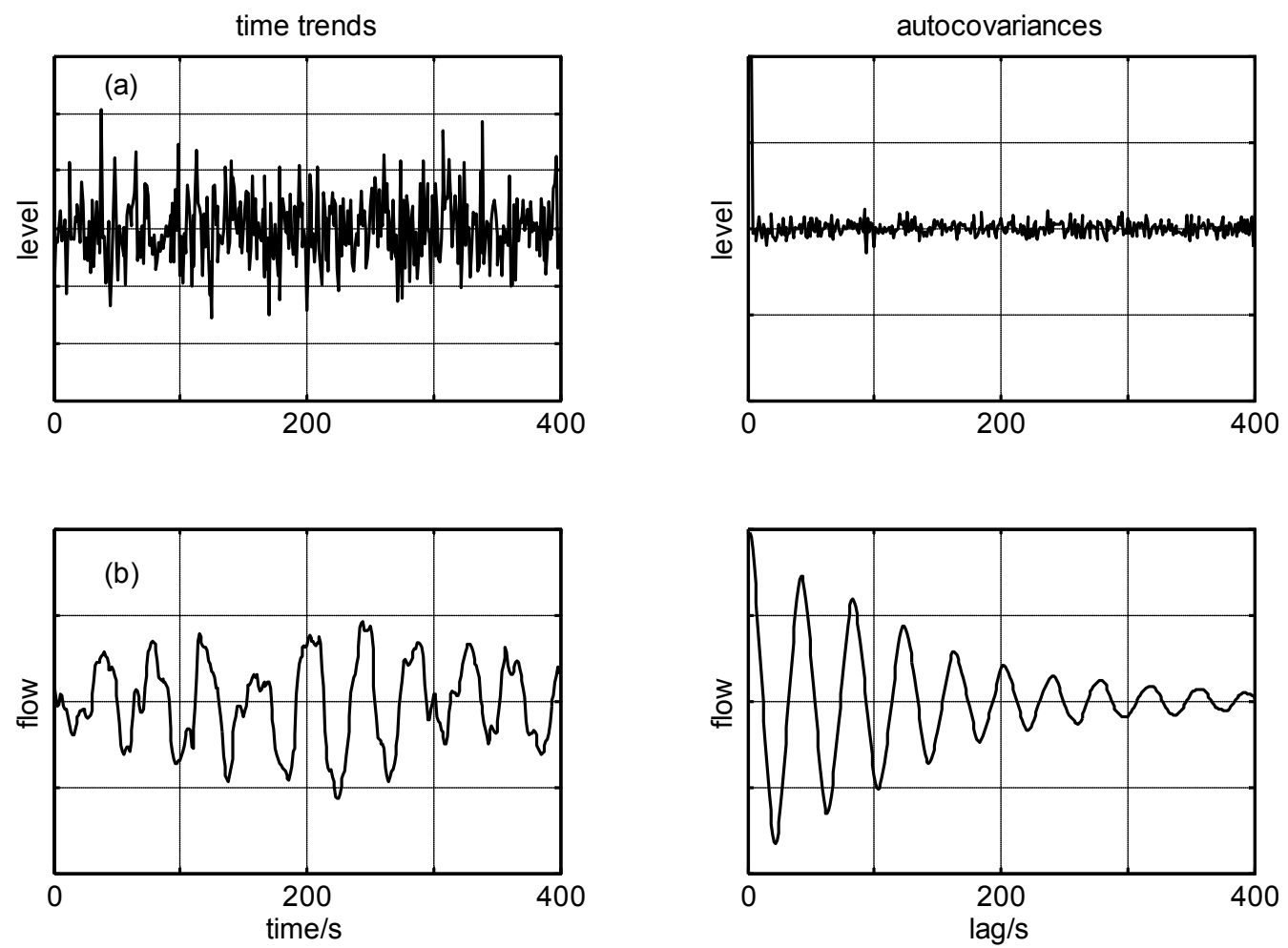

Figure. 4. Disturbance sequences captured from plant: (a) level disturbance from compressed air bubbles (b) cold water flow disturbance. The time trends are scaled to unit standard deviation and the autocovaince vertical axis is -1 to +1 . 

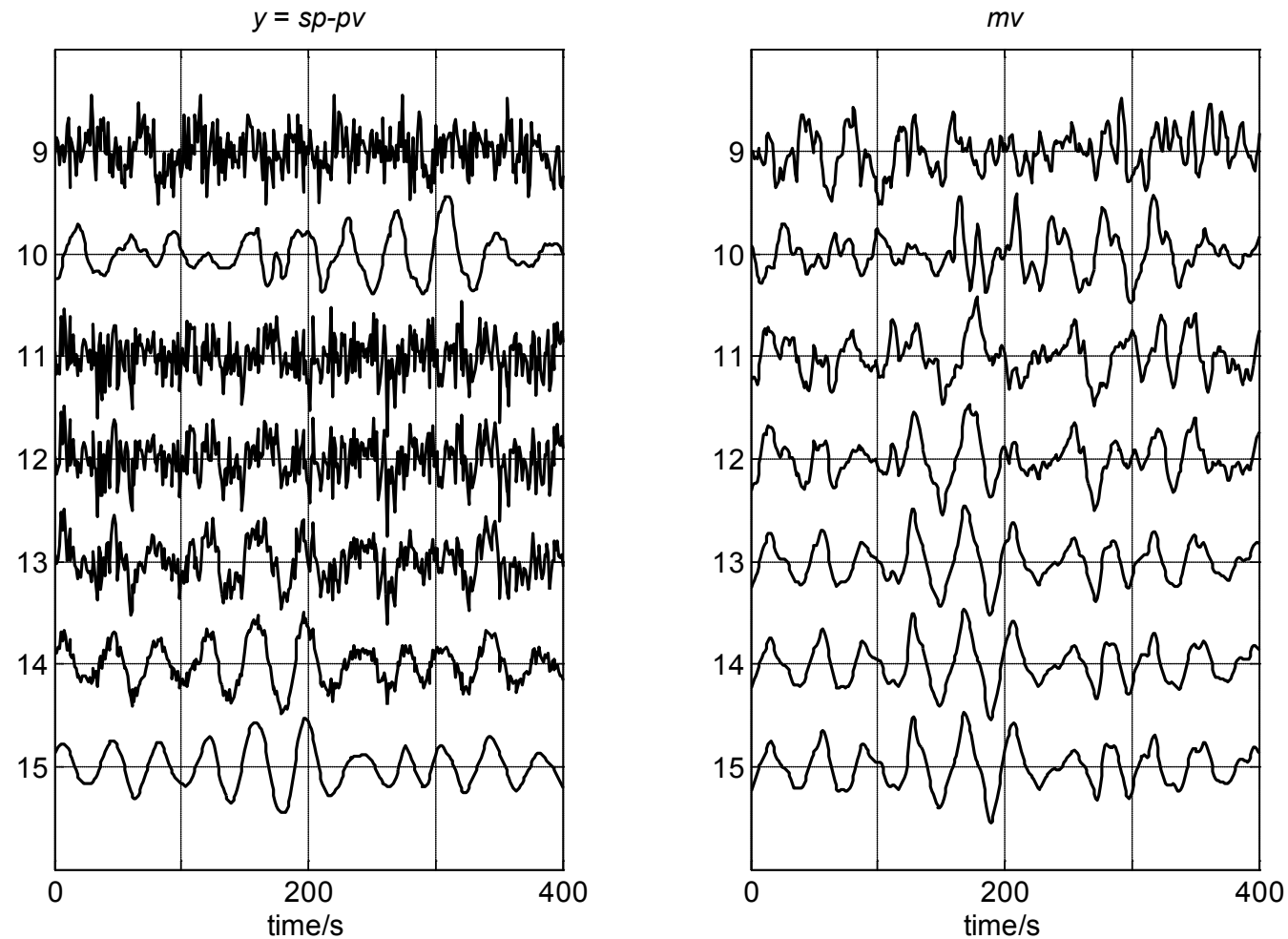

Figure 5. Pilot plant and simulation Tests 9 to 15 . Left panel: controller error trends; Right panel: $m v$ trends. The trends are normalised to unit standard deviation. 


\section{$y$, before}

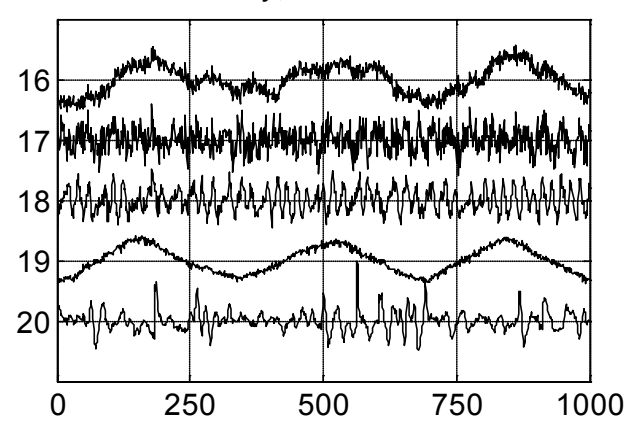

$y$, after

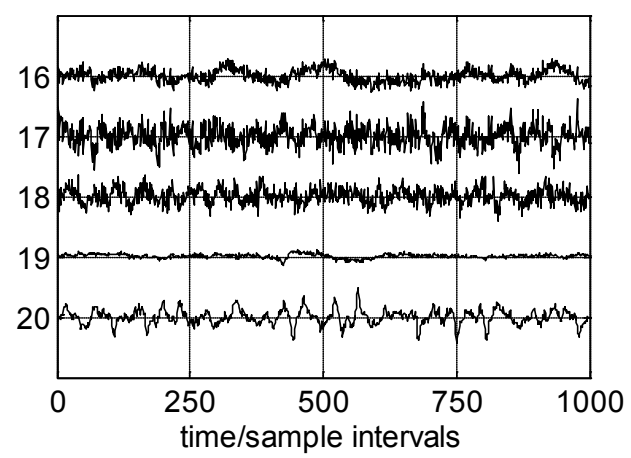

$m v$, before

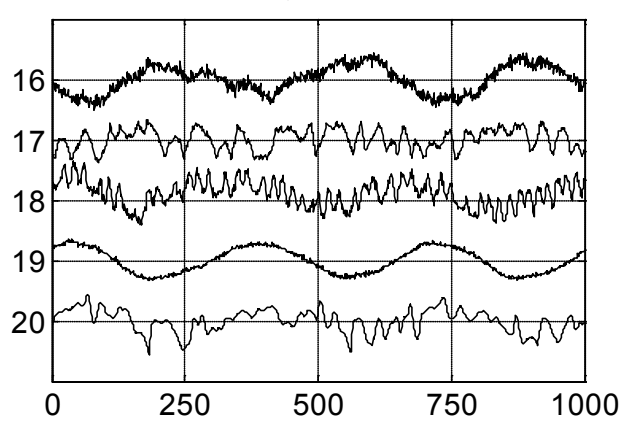

$m v$, after

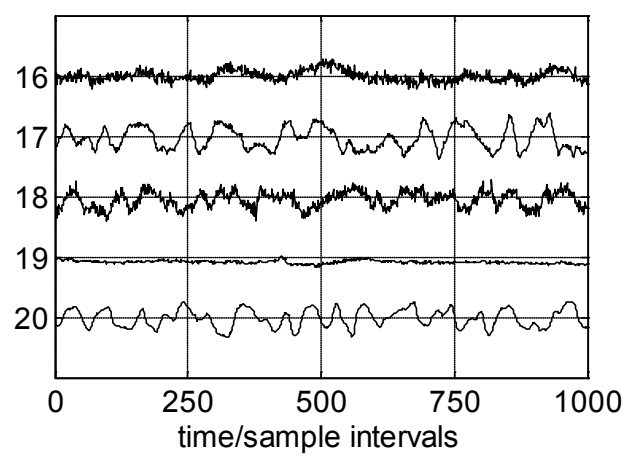

Figure 6: Industrial data from flow and level loops during a plant-wide disturbance. Upper panels: Plant-wide disturbance in controller error, $y$, and $m v$ caused by a sticking valve. Lower panels: The same measurements on the same vertical axis scales after the valve was repaired. 

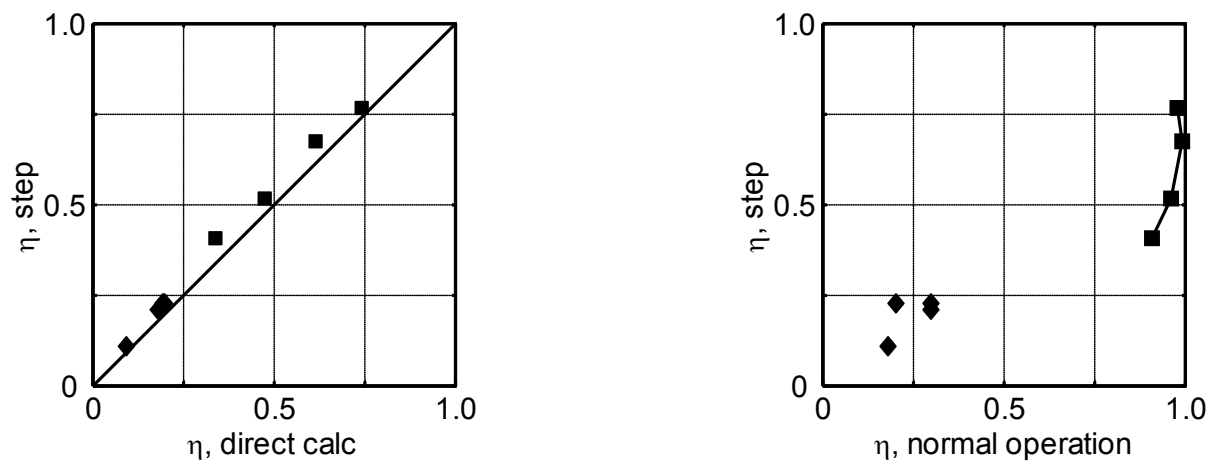

Figure 7. Performance index results for set point tracking during step testing and during regulatory operation: $(\diamond)$ cascade level loop, Tests 1 to $4(\boldsymbol{\square})$ temperature loop, Tests 5 to 8. 

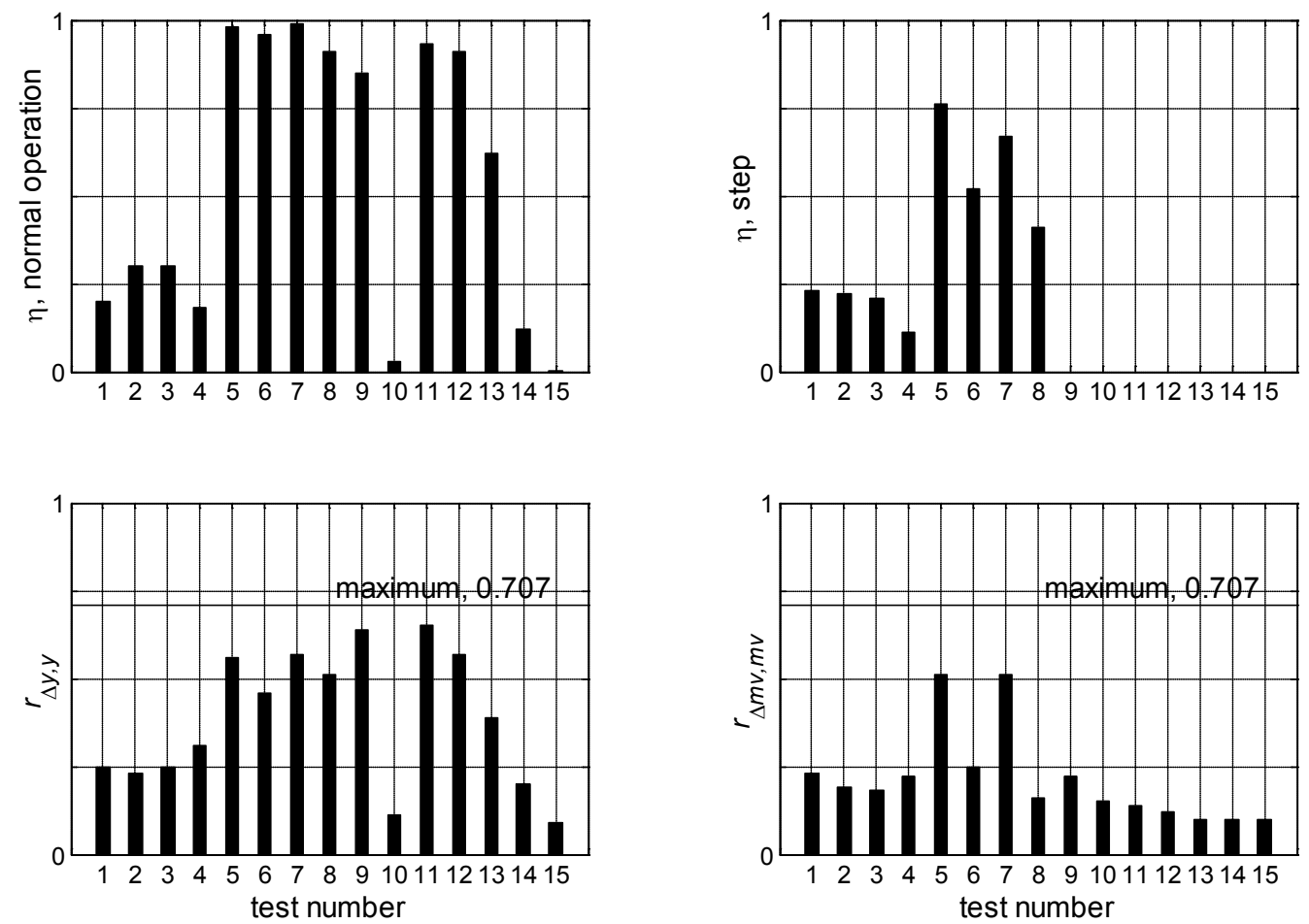

Figure 8. Graphical comparison of the performance index results during step tests and regulatory operation with the correlation coefficients $r_{\Delta y, y}$ and $r_{\Delta m v, m v}$. 


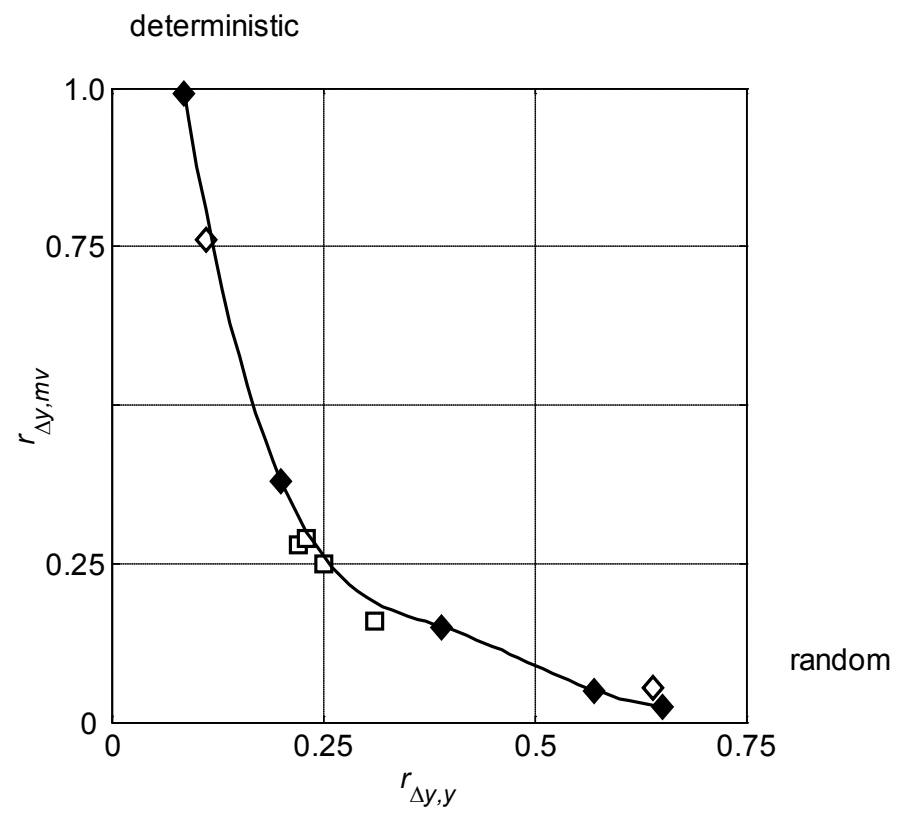

Figure 9. The relationship between correlation coefficients $r_{\Delta y, y}$ and $r_{\Delta y, m v}$ for the level loop: $(\bullet)$ simulation, Tests 11-15; $(\diamond)$ plant runs, Tests 9 and 10; $(\square)$ plant runs, Tests 1-4. 

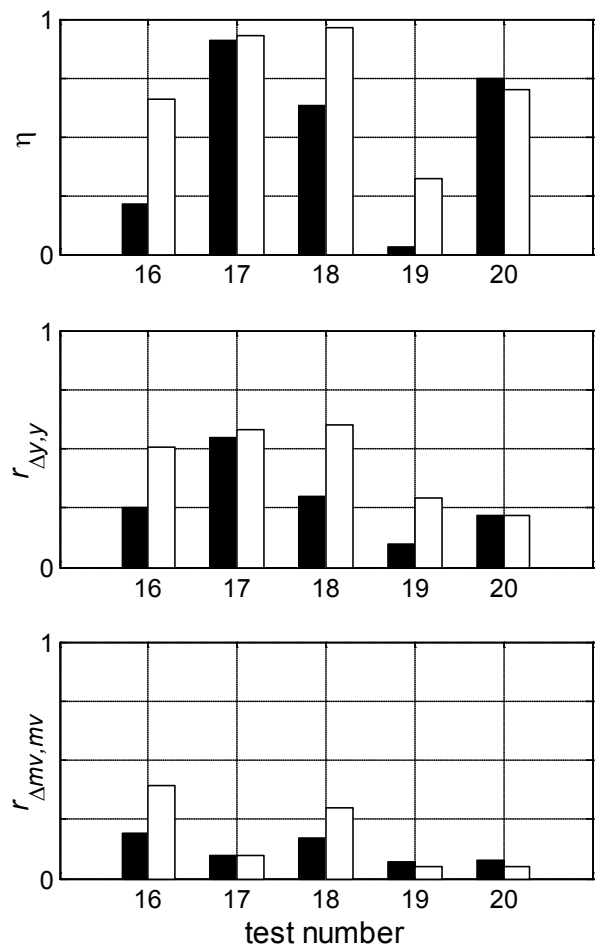

Figure 10. Graphical comparison of the performance indexes and correlation coefficients for the industrial data: Black bars: before repair of a faulty valve; White bars: after repair. 